\title{
When Coronary Artery Disease Meets a Channelopathy: An Unexpected Dangerous Date
}

\author{
Francielle Feitosa Dias-Santos ${ }^{a}$ Nicola Marziliano ${ }^{b-d}$ \\ ${ }^{a}$ Federal University of Sergipe, São Cristóvão, Brazil; ${ }^{b}$ Università degli Studi del Molise, Campobasso, Italy; ${ }^{\mathrm{C} C l i n i c a l}$ \\ Pathology Laboratory, ASL Taranto, Taranto, Italy; ${ }^{d}$ Fondazione Floresta Longo, Catania, Italy
}

A number of hereditary cardiac diseases are caused by autosomal dominantly inherited single-gene mutations (namely familial cardiomyopathies and channelopathies) [1]. Furthermore, coronary artery disease (CAD) - the leading global cause of mortality - has long been recognized to be heritable too and recent advances have started to unravel the genetic architecture of such a complex and multifactorial disease $[2,3]$.

As with most complex diseases, an individual's risk of developing CAD is modulated by an interplay between genetic and lifestyle factors. Clinical observations dating back to the 1950s have supported the notion that the risk for CAD is heritable [3]. A study of over 20,000 Swedish twins subsequently confirmed this finding of an increased risk for CAD among close relatives and estimated a heritability of $\approx 50 \%$ for fatal CAD. In 1985 , Lehrman et al. [4] first showed that a molecular defect in a single gene can drive CAD risk. Impaired receptor-mediated hepatic uptake of LDL leads to substantially increased levels of circulating cholesterol and premature CAD. Since then, many recent insights have led us to the understanding that the majority of the heritable risk for CAD is predicated on common variants with the exception of rare monogenic conditions such as familial hypercholesterol-

\section{KARGER}

() 2019 S. Karger AG, Basel

E-Mail karger@karger.com

www.karger.com/crd emia. These variants, in combination with environmental factors, lead to a quantitative blend of multiple driving processes in each individual.

Despite this knowledge on the etiology of CAD, little is known about complicating factors such as ventricular tachycardia and fibrillation (VT/VF) and its underlying genetic architecture. VT/VF are fatal arrhythmias that cause cardiac collapse and are major complications of acute myocardial infarction (AMI). In 1997, a panel of experts defined sudden death in the absence of an identifiable cause as idiopathic ventricular fibrillation (IVF): "IVF is the terminology that best acknowledges our current inability to identify a causal relationship between the clinical circumstance and the arrhythmia" [5]. In the same article, the requirements for the diagnosis of IVF were identified on the basis of the clinical tools available at the time. In 1992, using the phenotypes observed in the IVF registry, they advanced the hypothesis that IVF could be the manifestation of concealed forms of arrhythmogenic disorders [6] exacerbated by appropriate triggers and/or that it could be a consequence of them. A few years later, the discovery of the genes of long QT syndrome (LQTS) $[7,8]$ and the detection of incomplete penetrance supported the first hypothesis. 
Real-world data indicate that high-risk patients presenting with ventricular tachyarrhythmias and sudden cardiac arrest on admission reveal the highest long-term all-cause mortality in the absence of AMI compared with AMI patients [9]. But what if AMI occurs in individual mutation carriers of genes associated with channelopathies? Wang et al. [10] investigated this fatal coincidence in a cohort of 139 patients with AMI complicated with ventricular arrhythmias compared with 337 patients with AMI only. Of note, all of the individuals were of Chinese ancestry [11]. Previous data indicate important differences between Asian and white patients with a channelopathy such as Brugada syndrome. Among Asian patients, male adults are affected almost exclusively, more often with aborted cardiac arrest and spontaneous type $1 \mathrm{Bru}-$ gada syndrome-ECG. However, they have less family history of sudden cardiac death and markedly lower SCN5A mutation rates.

Thus, the paper by Wang et al. [10] can be considered an additional key stone in deciphering the phenotype presentation of channelopathies even in a subset of cases such as AMI. Namely, the authors explore the intriguing overlap between arrhythmogenic genes (such as SCN5A, $K C N Q 1$, and KCNH2 classically associated with LQT syndrome) and AMI. In particular, their aim is to explore the contribution of a possible arrhythmogenic substrate genetically determined within a frame of classical multifactorial disorder such as AMI.
The scientific work is well designed and conducted; data are presented in a robust way. The authors well explored the hypothesis of a genetic substrate underlying an excessive QT prolongation and life-threatening conditions in case of myocardial infarction. Indeed, in $8.6 \%$ of the cases they identified a disease-causing mutation in the AMI population, thus contributing to an increased risk of events. Their findings support the concept that the electrical remodeling associated with the healing phase of AMI may unmask a genetic substrate predisposing to a time-limited development of life-threatening arrhythmias. Additionally, these data represent the proof of concept that modifiers of the arrhythmic risk in a monogenic disease as LQTS may increase risk also in much more prevalent diseases, such as myocardial infarction.

\section{Acknowledgements}

N.M. is grateful to the Fondazione Floresta Longo (http://www. fla.it/fondazione) for the charity support to the patients and their families affected by channelopathies.

\section{Disclosure Statements}

F.F.D.-S. and N.M. have no conflicts to disclose neither financial commitments.

\section{References}

1 Tester DJ, Ackerman MJ. Genetic testing for potentially lethal, highly treatable inherited cardiomyopathies/channelopathies in clinical practice. Circulation. 2011 Mar;123(9): 1021-37.

2 Khera AV, Emdin CA, Drake I, Natarajan P, Bick AG, Cook NR, et al. Genetic Risk, Adherence to a Healthy Lifestyle, and Risk of Coronary Disease. N Engl J Med. 2016;375:2349-58.

3 Myocardial Infarction Genetics Consortium, Kathiresan S, Voight BF, Purcell S, Musunuru K, Ardissino D, Mannucci PM, et al. Genomewide association of early-onset myocardial infarction with single nucleotide polymorphisms and copy number variants. Nat Genet. 2009 Mar;41(3):334-41.

4 Lehrman MA, Schneider WJ, Südhof TC, Brown MS, Goldstein JL, Russell DW. Mutation in LDL receptor: Alu-Alu recombination deletes exons encoding transmembrane and cytoplasmic domains. Science. 1985 Jan; 227(4683):140-6.
5 Consensus Statement of the Joint Steering Committees of the Unexplained Cardiac Arrest Registry of Europe and of the Idiopathic Ventricular Fibrillation Registry of the United States. Survivors of out-of-hospital cardiac arrest with apparently normal heart. Need for definition and standardized clinical evaluation. Circulation. 1997 Jan;95(1): 265-72.

6 Priori SG, Borggrefe M, Camm AJ, Hauer RN, Klein $\mathrm{H}$, Kuck KH, et al. Unexplained cardiac arrest. The need for a prospective registry. Eur Heart J. 1992 Nov;13(11):1445-6.

7 Curran ME, Splawski I, Timothy KW, Vincent GM, Green ED, Keating MT. A molecular basis for cardiac arrhythmia: HERG mutations cause long QT syndrome. Cell. 1995 Mar;80(5):795-803.

8 Masuda M, Nakatani D, Hikoso S, Suna S, Usami M, Matsumoto S, et al.; OACIS investigators. Clinical Impact of Ventricular Tachycardia and/or Fibrillation During the Acute
Phase of Acute Myocardial Infarction on InHospital and 5-Year Mortality Rates in the Percutaneous Coronary Intervention Era. Circ J. 2016 Jun;80(7):1539-47.

9 Behnes M, Mashayekhi K, Weiß C, Nienaber C, Lang S, Reiser L, et al. Prognostic Impact of Acute Myocardial Infarction in Patients Presenting With Ventricular Tachyarrhythmias and Aborted Cardiac Arrest. J Am Heart Assoc. 2018 Oct; 7(19):e010004.

10 Wang F, Liu Y, Liao H, Xue Y, Zhan X, Fang $\mathrm{X}$, et al. Genetic variants on SCN5A, KCNQ1 and $\mathrm{KCNH} 2$ in patients with ventricular arrythmias during acute myocardial infarction in a Chinese population. Cardiology. 2019, doi: $10.1159 / 000502833$.

11 Milman A, Andorin A, Postema PG, Gourraud JB, Sacher F, Mabo P, et al. Ethnic differences in patients with Brugada syndrome and arrhythmic events: New insights from SABRUS. Heart Rhythm. 2019 Oct;16(10):146874. 